importance of combining the interests of both orthopaedic surgeons and preclinical researchers in one publication.

There has been a profound shift during recent decades from patient-orientated studies to research at the cellular and molecular level. Clinical research and audit in orthopaedics are time-consuming, and the most difficult and the slowest of all research, partly because of the long follow-up required. This has led to the increasing interest in basic research including such subjects as molecular biology and cytogenetics. These types of research are certainly important and necessary in orthopaedics, but need to be related to clinical problems.

For this reason, more orthopaedic surgeons are required with interests in research and in identifying clinical problems; the 'reductionist' researchers can then help with the necessary techniques. This calls for the combined efforts of orthopaedic surgeons and basic researchers. I see this cooperation symbolically as a nerve system, which starts from a clinical question, then sprouts into different branches of basic research, and eventually gathers the results together again for the benefit of our patients.

Some walls in Europe have already been torn down and borders have been opened. I hope that the EORS will be able to establish increasing co-operation in research networks, not only in Europe, but also between continents. The Journal of Bone and Joint Surgery will unquestionably provide the means of spreading information on musculoskeletal research throughout the world.

The EORS has selected an Editorial Board from various fields relevant to all aspects of musculoskeletal disease; its membership will be announced soon, and all will take an active part in the review process.

Our society is looking forward to this new co-operative venture.

LARS LIDGREN PRESIDENT EORS

\title{
Advice for authors - blinding and references
}

All journals and their editors conduct a balancing act between the overlapping groups which they try to serve. Authors would like 100\% acceptance; individual subscribers would like us to publish only good articles that will interest them personally. In the not-so-distant future, electronic means such as unlimited Internet memory and computer 'Knowbots' trained to make such individual selections, may be able to satisfy both groups (La Porte et al BMJ;310:1387-90). But at present one of our main tasks is the fair and efficient selection of the $16 \%$ of submissions that we are able to publish.

To increase the fairness of the selection process, we decided to start to blind our reviewers to the source of the submitted paper (editorial March 1996; 173-4). This has proved to be a satisfactory innovation and already approximately $70 \%$ of manuscripts arrive with a second cover page on which the names and addresses of the authors and the source of the work have been omitted. This is not quite enough; our astute reviewers often find clues in the text and in the references, on the back of illustrations and even on the envelopes containing them. In specialist subjects a reviewer may already be aware of the work, having heard it presented at a meeting. Blinding can rarely be perfect but it is designed to protect the author from prejudice and is in his interest; we hope for more and better blinded submissions, but we shall not return unblinded manuscripts.
The other change also announced in March 1996 and starting in our January 1997 issue is in our method of publishing references. We shall use the so-called 'Vancouver' system in which references in the text are numbered in the order of their appearance and the reference list at the end of the paper is in the order of these numbers. The main reasons for this change are the space taken up by strings of names and dates in the text, the increasingly specialist and scientific nature of published papers, and the wish to provide the best format for electronic presentation and publication.

The main objection, concerning which we have received just one letter, is that it will no longer be so easy to scan a reference list for a known first author or key paper. This seems a small price to pay for the improvements which will allow us to publish more papers and bring us into line with most scientific and general medical journals.

We will continue to review submitted papers in any reference format, but in future intend to return accepted papers for conversion to the correct format before editing begins. Our "Guide to Authors" has been amended, but it is well known to editors that this page of their journal is rarely studied.

PHILIP FULFORD EDITOR 